\section{Shave-Exzision mit herkömmlichen Rasierklingen}

T. Gambichler, S. Neuß, P. Altmeyer, K. Hoffmann

Klinik für Dermatologie und Allergologie

der Ruhr-Universität Bochum

(Direktor: Univ.-Prof. Dr. med. P. Altmeyer)
Zusammenfassung. Neben der spindelförmigen Exzision wird mit dem Skalpell häufig auch die tangentiale Exzision (Shave-Exzision) bei der Entfernung verschiedener Hauttumoren durchgeführt. Der Einsatz von herkömmlichen Rasierklingen bei der Shave-Exzision ist in Deutschland kaum verbreitet. Es können dafür handelsübliche, zweischneidige Rasierklingen eingesetzt werden, die extrem scharf sind und nach Sterilisation mehrmals verwendet werden können. Der Preis für eine Klinge liegt bei etwa 0,70 DM. Aufgrund der Flexibilität der Klinge lassen sich für Entfernung von oberflächlichen oder eher tiefer gelegenen Läsionen unterschiedlich konvexe Schnittflächen formen, was bei einer tangentialen Exzision von sehr großem Vorteil ist. Neben der oberflächlichen Abtragung von kosmetisch störenden Hautläsionen, wie z.B. seborrhoischen Keratosen und papillomatösen Nävi, eignet sich die Methode insbesondere für die Entfernung und histologische Untersuchung von makulären melanozytären Nävi, da man im Gegensatz zu einem starren Skalpell mit einer konvex gebogenen Rasierklinge auch tiefere, tangentiale Exzisionen leicht ausführen kann. Einerseits ist die histologische Auswertbarkeit von Exzisaten nach spindelförmigen Skalpell-Exzisionen und tiefen Shave-Exzisionen vergleichbar gut, andererseits sind die kosmetischen Ergebnisse nach Shave-Exzisionen in der Regel besser. Demnach ist die Shave-Exzision mit Rasierklingen eine kosmetisch günstige, kosteneffektive Methode insbesondere für Patienten, bei denen häufiger Hauttumoren (z.B. multiple Nävi) entfernt werden müssen.

Shave Excision with Common Razor Blades. Tangential excision (shave excision) is frequently performed with a scalpel for the removal of various skin tumors. In Germany, shave excision with common razor blades is not commonly employed. Doubleedged razor blades are readily available and extremely sharp. The price for one blade is about 0,70 DM; they can be re-used several times after sterilisation. Due to the flexibility of the blade, different convex cutting edges can be formed for the removal of superficial or deeper lesions. This is of great advantage for a tangential excision. The razor blade surgery is indicated for cosmetic excision of various lesions i.e. seborrhoic keratoses and papular nevi. Moreover, the technique is especially suitable for the removal and histologic examination of macular melano-

Akt Dermatol 2001; 27: 18-20

(c) Georg Thieme Verlag Stuttgart $\cdot$ New York ISSN 0340-2541 cytic nevi. In contrast to a inflexible scalpel, a convexly bowed razor blade allows deeper tangential excision. On one hand, the certainty of histologic diagnosis is comparable for elliptical excision and shave excision; on the other hand, better cosmetic results are usually obtained after shave excision. Thus, shave excision with razor blades is a cosmetically favorable, cost-effective method especially for patients who frequently undergo removal of skin tumors, i.e. multiple nevi.

\section{Einleitung}

Verschiedene Exzisionstechniken werden für die Entfernung und histologische Abklärung von Hautveränderungen eingesetzt. Sowohl die Stanzbiopsie und spindelförmige SkalpellExzision mit primärem Wundverschluss als auch die tangentiale Exzision (Shave-Exzision) mit sekundärer Wundheilung, die in der Regel auch mittels Skalpell durchgeführt werden, stellen etablierte dermatochirurgische Methoden dar. Eine in Deutschland wenig bekannte aber in den USA häufig eingesetzte Technik ist die Shave-Exzision mit herkömmlichen Rasierklingen $[1,2]$. Im Folgenden werden die Methoden und Indikationen der Shave-Exzision mit Rasierklingen kurz dargestellt und besprochen.

\section{Methoden und Indikationen}

Für die Shave-Exzision können handelsübliche, zweischneidige Rasierklingen (z.B. Gillette ${ }^{\circledR}$, Wilkinson ${ }^{\circledR}$ ) verwendet werden (Abb.1). Die Klingen sind extrem scharf und mit einem antikorrosiven Öl vorbehandelt, das auch bakteriostatische Substanzen enthält - demnach ist die Sterilisation einer neuen Klinge vor dem Eingriff nicht unbedingt nötig. Wie üblich wird die Haut mit einem Lokalanästhetikum betäubt. Die Kombination mit vasokonstriktorischen Substanzen (z.B. Adrenalin, Epinephrin) zur Reduktion intraoperativer Blutungen ist empfehlenswert. Insbesondere bei kleinen Hautveränderungen ist es von Vorteil, die Läsionen durch die Injektion des Lokalanästhetikums in Form einer Quaddel vom Hautniveau abzuheben.

Der seitliche Rand der Klinge wird zwischen Daumen und Mittelfinger gehalten. Aufgrund der hohen Flexibilität der Klinge kann durch mehr oder weniger starken Druck des Zeigefingers von oben auf das Zentrum der Klinge bei gleichzeitigem Anziehen der Klingenränder mit dem Daumen und Mittelfinger eine 


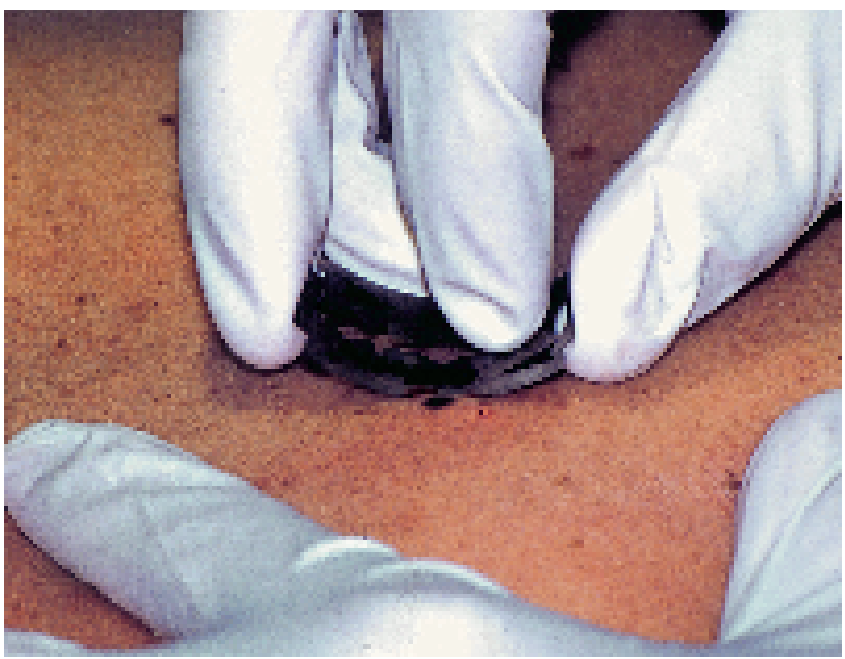

Abb.1 Shave-Exzision eines makulären melanozytären Nävus mit einer herkömmlichen doppelschneidigen Rasierklinge.

variabel konvexe Schnittfläche hergestellt werden. Dadurch lässt sich z.B. eine stark gebogene Schnittfläche formen, die bei kleinen oder tief gelegenen Hautveränderungen eine optimale Schnittführung und Eindringtiefe ermöglicht (Abb.1). Sowohl die Konvexität als auch der Winkel, in dem die Schnittfläche zur Hautoberfläche angesetzt wird, sind entscheidend für die Eindringtiefe der Rasierklinge. Nach dem Ansetzen der Klinge wird die Hautveränderung mit kleinen, seitlichen Schnittbewegungen aus dem Handgelenk (Radial- und Ulnarabduktion) exzidiert. Die andere Hand kann gleichzeitig das Operationsfeld z.B. durch Hautstraffung stabilisieren (Abb.1). Mit einem Wattestäbchen lässt sich das Exzisat während der Operation gestreckt fixieren (ggf. Assistenz), was insbesondere bei dünnen Hautproben von Vorteil ist [1]. Um das Einrollen des Exzisates im Formalinglas zu verhindern, kann es einfach nach dem Eingriff auf Filterpapier in gestreckter Form aufgebracht werden. Andere Methoden zur Fixation von Shave-Exzisaten wurden beschrieben $[1,3]$. Postoperativ werden häufig zur Hämostase chemische Substanzen (z.B. Eisen-III-Chlorid, Aluminium-Chlorid) eingesetzt. Bei der Shave-Exzision von pigmentierten Nävi wird zur Hämostase die Elektrokauterisation von manchen Autoren empfohlen. Die Wundpflege kann bei größeren und tieferen Shave-Exzisionen mit Wasserstoffperoxid oder einer antibiotischen Creme erfolgen [4-6]. Die Abheilung unter Bildung einer Kruste dauert etwa 7-14 Tage.

Die häufigsten kosmetischen Indikationen für eine Shave-Exzision sind papillomatöse Nävi und seborrhoische Keratosen, bei denen gewöhnlich nur eine oberflächliche Abtragung erforderlich ist $[2,7]$. Darüber hinaus ist die tiefe Shave-Exzision (retikuläre Dermis) mit einer Rasierklinge wegen ihrer großen Biegsamkeit besonders geeignet für die Entfernung und histologische Abklärung von makulären melanozytären Nävi - mit Ausnahme bei Verdacht auf Malignität $[1,2,4,7,8]$. Weitere Indikationen wurden berichtet, wie z. B. epidermale Nävi, Rhinophym, Granuloma pyogenicum, Spalthautproben, aktinische Keratosen und oberflächliche Basalzellkarzinome [2,9,11].

\section{Diskussion}

Die Shave-Exzision mit herkömmlichen Rasierklingen ist eine einfache, schnelle und kostengünstige Operationsmethode. Der Preis für eine zweischneidige Rasierklinge liegt bei ca. 0,70 DM, wobei die Klingen in der Regel mindestens zweimal verwendet werden können. Die Unhandlichkeit der Rasierklingen ist gewöhnungsbedürftig. Um die unbeabsichtigte Verletzungsgefahr von Patient und Arzt zu reduzieren, werden mehrere Hilfsmittel empfohlen. Der Rasierklingen-Halter nach Castroviejo fixiert eine viertel Klinge (längs halbiert, quer geteilt) in einer Griffvorrichtung. Das Zerbrechen oder Zerschneiden der Klinge birgt aber wiederum Verletzungsgefahren in sich [12]. Der größte Nachteil dieser Methode ist, dass die Klinge nicht mehr in gebogener Form flexibel eingesetzt werden kann und somit eine kontrollierte tangentiale Exzision von makulären Läsionen unmöglich ist. Die Verwendung eines steril verpackten Polyethylen-Adaptors bewahrt die Flexibilität der Klinge, bietet mehr Schutz vor Verletzungen und gibt der Klinge ein professionelleres Erscheinungsbild. Der in den USA erhältliche Einmal-Adapter kostet etwa 1 DM [13]. Basierend auf eigenen Erfahrungen bevorzugen wir zur Shave-Exzision die Verwendung der zweischneidigen Rasierklinge ohne zusätzliche Hilfsmittel. Bei entsprechend vorsichtiger Handhabung halten wir das Verletzungsrisiko vergleichbar mit dem Umgang von Skalpell und Spritzenkanülen [2,14].

Im Vergleich zum Skalpell lässt sich mit der Rasierklinge sowohl kostengünstiger operieren als auch wesentlich besser eine Shave-Exzision von makulären Läsionen wie z. B. melanozytären Nävi durchführen. Eine tangentiale Exzision von kleineren Läsionen kann zwar auch mit sehr scharfen Ringküretten vorgenommen werden, aber die Kosten für den Eingriff sind höher als mit einer Rasierklinge, zumal für unterschiedlich große Nävi auch verschieden große Küretten bereitgehalten werden müssten. Eine tiefe Shave-Exzision mit einem Skalpell oder einer Rasierklinge bringt histologisch gut beurteilbare Präparate, die mit denen von spindelförmigen Skalpell-Exzisionen vergleichbar sind. Im Gegensatz dazu treten bei Stanzbiopsaten häufig größere diagnostische Unsicherheiten auf. Demnach können auch atypische melanozytäre Nävi mittels Shave-Exzision entfernt werden $[1,2,15]$. Eine vorherige dermatoskopische Untersuchung ist empfehlenswert. Bei Verdacht auf Malignität sollte selbstverständlich eine spindelförmige Skalpell-Exzision erfolgen. Die Rezidivrate nach ShaveExzisionen mit Rasierklinge oder Skalpell wird in der Literatur mit $2-18 \%$ angegeben $[1,4,16]$. Bei einer Auswertung nachexzidierter atypischer melanozytärer Nävi wurden Nävusreste signifikant häufiger nach Stanzexzisionen als nach Shave-Exzisionen und spindelförmigen Skalpell-Exzisionen histologisch beobachtet [8]. Sporadisch kommt es bei inkompletter Exzision von melanozytären Nävi zum Auftreten von „Pseudomelanomen“, die sowohl klinisch als auch histologisch Kriterien maligner Entartung aufweisen, so dass insbesondere für den unerfahrenen Untersucher diagnostische Schwierigkeiten auftreten können (Abb. 2). Von zentraler Bedeutung ist für den Untersucher einer solchen Läsion die Kenntnis, dass es sich um ein Rezidiv nach Exzision eines melanozytären Nävus handelt $[1,17]$.

Die kosmetischen Ergebnisse nach Shave-Exzision sind in der Regel besser als nach spindelförmiger Skalpell-Exzision, bei der die gesamte Dermis durchtrennt wird und häufig dehäs- 


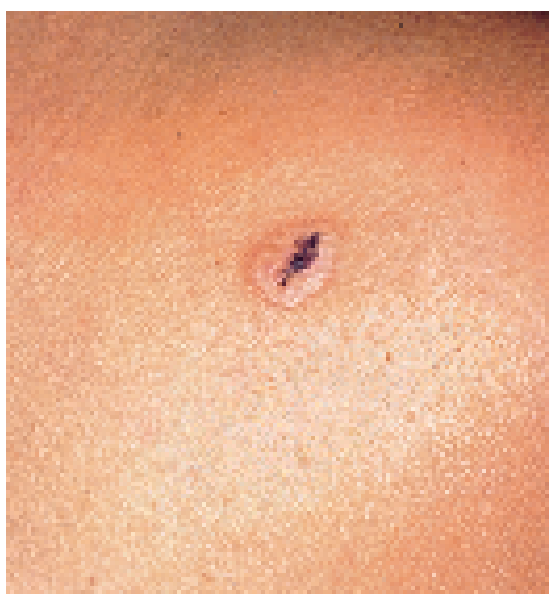
Nävus nach ShaveExzision in Form eines „Pseudomelanoms“.

Abb. 3 Leichte

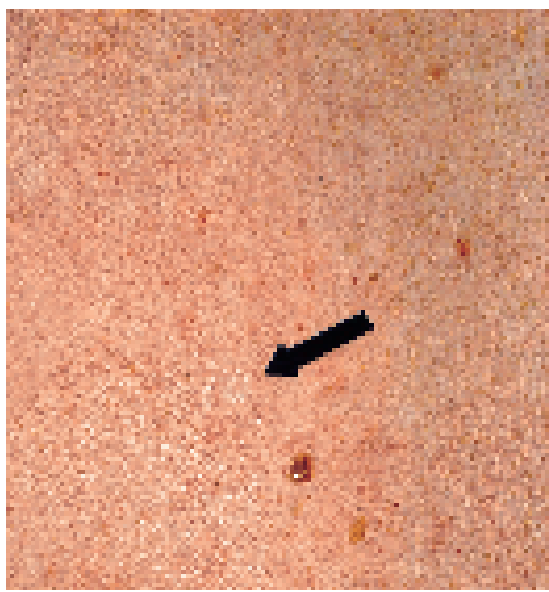
Hypopigmentierung (Pfeil) 6 Monate nach Shave-Exzision eines melanozytären Nävus am Rücken mit Rasierklinge.
Abb. 2 Rezidiv-

zente Narben entstehen $[1,2,4,5,18,19]$. Insbesondere bei einfachen Nahttechniken entstehen oft zusätzlich unschöne Narben im Bereich der Nahteinstiche. Bei einer Nachbeobachtung von Patienten, die sich einer Nävus-Exzision mittels Rasierklinge unterzogen, wurden nach 6 Monaten vorwiegend leichte Hypopigmentierungen bzw. Hyperpigmentierungen in den Exzisionsstellen gesehen (Abb. 2 u. 3). Hypertrophe und atrophe Narbenbildungen wurden nur selten beobachtet [4]. Insbesondere für Patienten mit multiplen melanozytären Nävi, dysplastischem Nävus-Syndrom und multiplen Rumpfhautbasaliomen, bei denen häufig Exzisionen vorgenommen werden müssen, stellt die Shave-Exzision mit herkömmlichen Rasierklingen eine kosteneffektive operative Methode mit guten kosmetischen Ergebnissen dar.

\section{Literatur}

${ }^{1}$ Haneke E. Flachexzision von Nävuszellnävi. In: Garbe C, Rassner G (Hrsg.). Leitlinien und Qualitätssicherung für Diagnostik und Therapie. Berlin, Heidelberg: Springer, 1998: 523-525

${ }^{2}$ Grabski WJ, Salasche SJ, Mulvaney MJ. Razor-blade surgery. J Dermatol Surg Oncol 1990; 16: 1121 - 1126

${ }^{3}$ Drummond CC, Hurwitz RM. Flat fixation technique for shave biopsy specimens: stick \& shake. J Dermatol Surg Oncol 1988; 14: $38-40$

${ }^{4}$ Gambichler T, Senger E, Rapp S, Alamouti D, Altmeyer P, Hoffmann K. Deep shave excision of macular melanocytic nevi with the razor blade biopsy technique. Dermatol Surg 2000; 26 : $662-666$

${ }^{5}$ Hudson-Peacock MJ, Lawrence CM. Cosmetic outcome following shave excision of benign papular naevi using either electrocautery or aluminium chloride for haemostasis. Br J Dermatol 1995; 133 (Suppl): 47

${ }^{6}$ Phillips T, Kapoor V, Provan A, Ellerin T. A randomized prospective study of a hydroactive dressing vs conventional treatment after shave biopsy excision. Arch Dermatol 1993; 129: 859-860

${ }^{7}$ Porter JM, Treasure J. Excision of benign pigmented skin tumours by deep shaving. Br J Plast Surg 1993; 46: 255-257

${ }^{8}$ Cohen LM, Hodge SJ, Owen LG, Callen JP. Atypical melanocytic nevi. Clinical and histopathologic predictors of residual tumor at reexcision. J Am Acad Dermatol 1992; 27: 701 - 706

${ }^{9}$ Harrison PV. Therapy of basal cell carcinoma: treatment in $180 / 81$ compared with 1985/86. An advantage use of shave excision for smaller tumours. Br J Dermatol 1987; 117: 349- 357

${ }^{10}$ Kirschner RE, Low DW. Treatment of pyogenic granuloma by shave excision and laser photocoagulation. Plast Reconstr Surg 1999; 104: 1346 - 1349

11 Zuber TJ, Jackson E. Chondrodermatitis nodularis chronica helicis. Arch Fam Med 1999; 8: 445-447

${ }^{12}$ Lum D, Lum R. Razor blade surgery: use of the Castroviejo blade breaker and holder. Dermatol Surg 1999; 25: 143 - 144

${ }^{13}$ Harvey DT, Fenske NA. The razor blade biopsy technique. Introduction of the adaptor-designed shave biopsy instrument. Dermatol Surg 1995; 21: $345-347$

${ }^{14}$ Field LM. Improving razor blade biopsy techniques. Dermatol Surg 1996; 22: $482-483$

15 Pariser RJ, Divers A, Nassar A. The relationship between biopsy technique and uncertainty in the histopathologic diagnosis of melanoma. Dermatol Online J 1999; 5: 4

${ }^{16}$ Breuninger H, Garbe C, Rassner G. Shave-Exzision von melanozytären Nävi der Haut. Hautarzt 2000; 51: 575-580

17 Kornberg R, Ackerman AB. Pseudomelanoma: recurrent melanocytic nevus following partial surgical removing. Arch Dermatol 1975; $111: 1588$ - 1590

${ }^{18}$ Hudson-Peacock MJ, Bishop J, Lawrence CM. Shave excision of benign papular naevocytic naevi. Br J Plast Surg 1995; 48: 318 - 322

${ }^{19}$ Harrison PV. Good results after shave excision of benign moles. J Dermatol Surg Oncol 1985; 11: 668

\section{Dr. med. Thilo Gambichler}

Klinik für Dermatologie und Allergologie

Ruhr-Universität Bochum

Gudrunstraße 56

44791 Bochum

E-mail: T.Gambichler@derma.de 